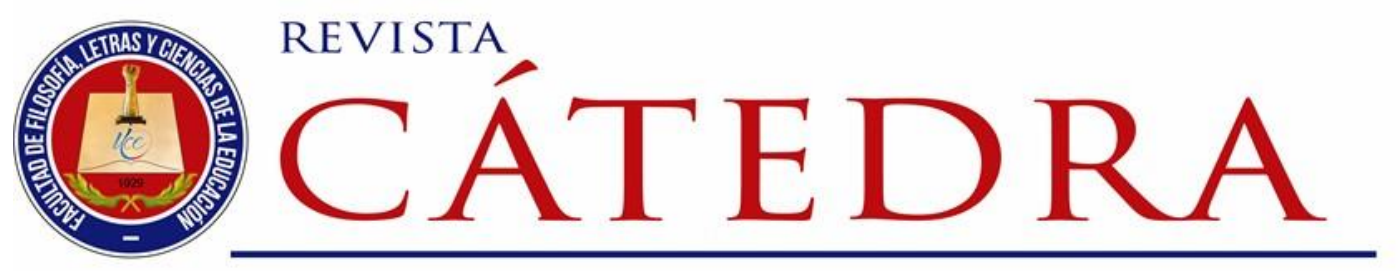

\title{
Las competencias digitales en docentes y futuros profesionales de la Universidad Central del Ecuador
}

\section{Digital competences in professors and students of Universidad Central del Ecuador}

\author{
Juan Cobos-Velasco \\ Universidad de Central del Ecuador, Quito,Ecuador \\ jccobos@uce.edu.ec \\ https://orcid.org/0000-0002-9770-3727 \\ Lilian Mercedes Jaramillo-Naranjo \\ Universidad UTE, Quito, Ecuador \\ lilian.jaramillo@ute.edu.ec \\ http://orcid.org/0000-0002-0586-4292 \\ Santiago Vinueza- Vinueza \\ Universidad Central del Ecuador, Quito, Ecuador \\ sfvinueza@uce.edu.ec \\ http://orcid.org/0000-0002-0818-6554
}

(Recibido: 20/05/2018; Aceptado: 1/06/2018; Versión final recibida: 15/06/2018)

Cita del artículo: Jaramillo-Naranjo, L., Cobos-Velasco, J. y Vinueza-Vinueza, S. (2019). Las competencias digitales en docentes y futuros profesionales de la Universidad Central del Ecuador. Revista Cátedra. 2(1),76-97.

\section{Resumen}

En la actualidad, el rol que desempeñan los docentes de educación superior se fortalece con el manejo de la conectividad, ya que para enseñar a sus estudiantes requieren del conocimiento y aplicación de herramientas tecnológicas que ofrece la web 2.0. Por lo que los docentes deben aprestarse no solo a adquirir conocimientos básicos en tecnología, sino a ser competentes para aplicar ésta en sus prácticas didácticas y, para lograrlo, su enseñanza aprendizaje inicial resulta esencial. El objetivo de esta investigación es realizar una aproximación diagnóstica sobre las competencias digitales que poseen futuros profesionales y docentes en el ejercicio de su profesión. Para ello, de una población total de 
40.000 estudiantes de la Universidad Central del Ecuador se extrajo la muestra óptima de 1.799, quienes respondieron a un cuestionario de percepción de conocimiento, aplicación y valoración de competencias digitales. Según los resultados, la mayor parte de los futuros profesionales disponen de un nivel básico de competencia digital. En el caso de los docentes se determinó que el uso de las herramientas de la web 2.0 causa dificultad en la incorporación a sus procesos de enseñanza, mientras que los estudiantes tienen un apego al uso de las mismas. La implementación de recursos tecnológicos en el proceso de enseñanza- aprendizaje, promueve un cambio significativo en instituciones de educación superior, esto permitirá formar profesionales capaces de enfrentar la sociedadactual.

Palabras clave

Competencia, digital, docentes, estudiantes, educación, TIC.

\section{Abstract}

The current role of professors working at the University strengthen due to the connectivity, since in order to teach the students the professors need to know the different technological tools offered by the Web 2.0 through Information Technology and Communication-ICT, reason for which professors must be prepared not only to acquire basic knowledge in technology, but to be competent to apply this information in their teaching practices. The objective of this research is to know the digital skills of future professionals based on their perception. For this, 1,799 out of a total population of 40,000 students of Universidad Central del Ecuador answered a questionnaire on knowledge perception, application and assessment of digital competences. According to the results, most of the future professionals have a high level of digital competence (especially in basic digital skills); likewise, certain significant contrasts were obtained with respect to age in the area of basic digital skills. In the case of teachers, it was determined that the use of web 2.0 tools causes difficulty in teaching processes, while students have an attachment in their use. The implementation of technological resources in the teaching-learning process promotes a significant change in institutions of higher education, which will allow to train professionals capable of facing today's society.

\section{Keywords}

Digital, skills, teachers, students, education, TIC.

\section{Introducción}

Esta investigación tiene como propósito fundamental identificar las dificultades que tienen docentes y estudiantes de la Universidad Central del Ecuador en el desarrollo de las competencias digitales y la incorporación de las Tecnologías de la Información y Comunicación (TIC) en el proceso de enseñanza- aprendizaje. La educación superior requiere suplantar metodologías tradicionales con la incorporación de las TIC para enfrentar los nuevos retos del siglo XXI.

Desde este enfoque varias han sido las exigencias para la educación superior: entre ellas la incorporación de las TIC; competencia tecnológica encargada de desarrollar habilidades integrales en estudiantes y docentes. La agenda 2030 tratada por la Organización de las Naciones Unidas (ONU) indica que "la educación superior tiene la obligación de encaminarse a promover no solo las competencias digitales, sino las competencias humanas y no únicamente sapiencias cerradas o técnicas programadas" (Pozo y Monereo, 1999, p. 
11). Esto involucra que se desarrollen el aprender a aprender y el aprender a ser persona, consideradores pilares de la educación del siglo XXI.

En tal virtud, para potenciar el desarrollo de las competencias digitales y que se fortalezca lo señalado por la ONU en la agenda 2030 es necesario el desarrollo autónomo y colaborativo de docentes y estudiantes. Los estudiantes requieren autorregularse para aprender a tomar decisiones y solucionar problemas en condiciones de conflicto e incertidumbre en su contexto diario. Los docentes requieren desarrollar procesos de aprendizaje para transformar conocimientos cognitivos en saberes prácticos y duraderos.

En concordancia con lo expuesto "la sociedad del conocimiento, las tecnologías de la información, los multimedia y las telecomunicaciones otorgarán a su profesión nuevos significados y roles" (Latapí, 2003, p. 15). En este marco de nuevas demandas que se plantean a los futuros profesionales, las instituciones educativas superiores se han visto en la necesidad de incorporar las TIC en los distintos escenarios educativos para mejorar los procesos de formación de futuros profesionales. Todo aquello involucra cambios en los modelos educativos que se ajusten a rediseños de carreras, cambios que sean factibles y brinden posibilidades metodológicas a los sistemas de enseñanza-aprendizaje.

Bajo esta perspectiva de cambio, docentes y estudiantes enfrentan planes institucionales solitarios, desactualizados, metodologías tradicionalistas y pensamientos lineales de docentes que se resisten a utilizar la tecnología. El presente estudio pretende analizar la percepción de docentes y estudiantes acerca de la competencia digital.

Para ese propósito se diseñó, se validó y se administró un cuestionario de competencias digitales para docentes en el que se evaluó el uso de plataformas educativas. Los hallazgos obtenidos son inspiradores y permiten apreciar algunas fortalezas y carencias formativas de los actuales planes de estudio y replantear nuevas estrategias para su correcto desarrollo. Las preguntas de investigación que guían el estudio son las siguientes:

- ¿Cuál es el nivel de competencias digitales que maneja el docente para potenciar el uso de la plataforma virtual de la Universidad Central del Ecuador?

- ¿Con qué frecuencia usa tecnología el profesorado de la Universidad Central del Ecuador?

- ¿Qué herramientas de la web 2.0 permiten reforzar el aprendizaje áulico y su implementación en la plataforma educativa universitaria?

- ¿Qué elementos estructurales y funcionales de la plataforma virtual mejorarán la enseñanza y fortalecerán el trabajo autónomo de los estudiantes?

- ¿Cuál es el nivel de conocimiento sobre las TIC que reportan los profesores de la Universidad Central del Ecuador?

Las preguntas planteadas se resolverán a través de la investigación cuantitativa, cuyo contexto se centra en los docentes y estudiantes de la Universidad Central del Ecuador. La población objeto de estudio de la investigación estuvo constituida por 1.197 docentes, y se tomó como muestra óptima a 300 profesores; la población estudiantil fue de 40.000 estudiantes, y se tomó como muestra óptima a 1.799.

Para responder cada interrogante conviene ubicarnos en el marco de los procesos de innovación, determinando la relación tecnología-educación: "innovación tecnológica, tendencia a la universalización y globalización, cambios en las relaciones sociales y nuevas concepciones de las relaciones tecnología-sociedad y tecnología-educación" (Cabero, Martínez, Salinas, 2003, p. 30). Con esta apreciación se puede determinar que no se debe quedar al margen del incremento de las nuevas TIC, por dos ámbitos muy importantes: el 
primero, los medios configuran una sociedad nueva en donde el sistema educativo tiene que servir; el segundo, el sistema educativo siempre va a utilizar los medios en la comunicación social, hoy en día esto sucede por el uso de las redes de telecomunicación.

Si bien la educación no debe quedarse al margen del avance de la tecnología, es de vital importancia que el aprendizaje y el conocimiento que se obtiene sea trasladado a la transformación de métodos y técnicas pedagógicas. Es así que, los objetivos educativos deben estar bien definidos y claros para que el estudiante se adapte a la nueva sociedad cambiante y globalizadora.

El artículo está estructurado en cinco partes: la primera parte presenta generalidades sobre qué significa cambio educativo y cómo las TIC se convierten en el material digital para mejorar aprendizajes autónomos y colaborativos fortalecidos de la Web 2.0. La segunda parte presenta varias consideraciones científicas sobre teorías fundamentados de varios autores que fortalecen el empleo de las TIC en escenarios educativos modernos, con la posibilidad de formar docentes que se alineen al paradigma de la conectividad y lenguajes tecnológicos. La tercera parte estudia conceptos relacionadosal desarrollo de competencias de las TIC para la alfabetización digital. La cuarta parte presenta los instrumentos de recolección de datos y sus resultados. La quinta parte, analiza los resultados obtenidos y las conclusiones.

\section{Generalidades sobre el contexto en el cambio educativo}

En la investigación realizada por Sabater (1997), sobre las nuevas funciones docentes y los nuevos medios como poderosos distribuidores de información, indica que se requiere un cambio en las funciones docentes por excelencia, esto es, la transferencia de los contenidos explicativos. Es así que en la actualidad a los docentes les resulta difícil competir con estos nuevos soportes en su extensión explicativa, ya que el rol del docente va más allá. La idea de que el docente es una fuente de información es una realidad a medias "son fuente de formación, no de información" (Savater 1997, pág. 45). Alonso en el (2001) también señala "Uno puede informarse dándole a un botón, lo que no puede es educarse dándole a un botón" (pág. 57). La nueva sociedad de la información espera que el docente aprenda a trasmitir nuevos conocimientos de forma diferente, es decir, espera que los nuevos medios den las herramientas que ayuden a olvidar viejos métodos que se utilizan en los procesos de enseñanza-aprendizaje.

Bajo la perspectiva de cambio de los docentes, los autores asumen que el profesorado no está capacitado para enfrentarse al cambio o simplemente están llenos de prejuicios. La tecnología resulta ser abrumadora, en especial para docentes que pertenecen a una escuela de enseñanza tradicionalista. La nueva sociedad aspira que los docentes, especialmente de educación superior, se conviertan en mediadores capaces de adaptarse y acogerse a los nuevos acontecimientos que surjan con la incorporación de las TIC.

Actualmente, las TIC han sido denominadas como la sociedad de la información, de la transformación. La transformación no solo en el campo político y social, sino también educativo. En el análisis de la obra de Duderstand (1997) se puede visualizar cuatro significativos argumentos para un mejor entendimiento del nuevo cambio social:

1. Es fundamental y de gran importancia el conocimiento para establecer la seguridad, bienestar y calidad de vida; frente a estos aspectos se tiende a obtener respuestas institucionales de distinto tipo.

2. Incorporar las TIC en el proceso de enseñanza-aprendizaje mediante programas de innovación docente en las diferentes instituciones de educación superior. 
3. Al momento que las tecnologías empiezan a ser consideradas en el organigrama y en los órganos de gestión de las universidades deben transformar las estructuras universitarias.

4. Relación con el aprovechamiento de las posibilidades comunicativas de las TIC en la docencia universitaria a través de experiencias motivacionales e innovadoras de todo tipo.

Todos estos elementos encaminan a la creación de nuevos procesos de enseñanzaaprendizaje. Es importante en este proceso, también desarrollar diferentes niveles de observación a las destrezas emocionales e intelectuales. En un mundo vertiginoso e invariable del cambio, la predisposición y el compromiso de los estudiantes debe ser fundamental y de gran importancia; "la flexibilidad de los alumnos para entrar en un mundo laboral que demandará formación a lo largo de toda la vida; y las competencias necesarias para este proceso de aprendizaje continuo" (Salinas, 1997, pág. 3).

El aprendizaje, según se entiende depende del entorno en el que el estudiante se encuentre. Por ejemplo, si se requiere desarrollar una página web, el estudiante debe tener al alcance las herramientas necesarias para su desarrollo. Los recursos que se utilizan en la enseñanza son los principales componentes curriculares que, por sus procedimientos simbólicos y estrategias ayudan al progreso de habilidades cognitivas en las personas. Captar y comprender la información por parte del estudiante permite la creación de ambientes especiales en los cuales se propician los escenarios de aprendizaje, incluyendo dos componentes fundamentales: el primero es el componente físico como el hardware. El segundo es el componente intangible o sistema software, procediendo estos dos aspectos a un contexto específico de comunicación educativa, con el principal objeto de proporcionar el aprendizaje (Cabero et al., 1999, pág. 53).

De igual manera, el ambiente en el que trabaja el docente al utilizar tecnología cambia de manera fructífera, es decir, el docente se convierte en un guía activo incentivando a los estudiantes a la utilización de los recursos digitales. Las herramientas son fundamentales para investigar y transformar conocimientos nuevos y destrezas; pasando a conducirse como gestor de la generación de nuevos recursos de aprendizaje, fortaleciendo su papel de guía y mediador (Salinas, 1998, pág.5-15).

\section{Cambios en la educación superior y TIC}

\subsection{Usos de los medios y tecnologías en la enseñanza}

Cuando el estudiante utiliza los materiales multimedia puede observar, explorar, experimentar $y$, por ende, es quien por iniciativa propia se da cuenta de sus errores, por ejemplo, al interactuar con una simulación. De acuerdo al análisis de la obra Materiales curriculares con Parcerisa (1996) los medios y tecnologías de la información se establecen de la siguiente manera:

- Innovadora: se incorpora materiales en la enseñanza que permiten realizar cambios estructurales e innovadores, ya que en varias instituciones educativas los materiales que se utilizan no son de gran innovación, sino más bien son rutinarios en el proceso de enseñanza aprendizaje, las situaciones son provocadas por la falta de conocimiento, habilidad para el correcto manejo de los mismos, poca convicción auténtica por los medios tecnológicos de la información, etc.

- Motivadora: se establecen estrategias en las cuales el interés y la atención de los estudiantes se convierten en actividades significativas y principalmente sugerentes, ya que el uso inadecuado de estos medios provoca una desmotivación en los 
estudiantes; es así que, en el aula de clases, la utilización de estos medios tiene que ver más con la pérdida de tiempo que con el establecer una planificación verdaderamente significativa.

- Configuradora y mediadora: los recursos que el estudiante utiliza para su autoaprendizaje determinan el tipo de actividad mental que los mismos desarrollan, la ventaja que se adquiere es generar nuevos conocimientos, los cuales pueden ser combinados para que de esta manera se conviertan en un aprendizaje significativo.

- Controladora: la información y los contenidos que se van a enseñar, si bien es cierto que se encuentran en la web en ocasiones resulta errónea o es permanentemente volátil, abierta o dinámica, razón principal por la cual las destrezas que desarrollan los alumnos no son relevantes ni suficientes. Una manera de estimular el proceso de enseñanza aprendizaje es por medio del aspecto lúdico ya que se pueden realizar juegos con contenidos que tengan relevancia para quien juega.

- Solicitadora: hoy en día se presentan nuevas formas de utilizar la información que requieren de habilidades intelectuales nuevas, las cuales todavía no son implementadas en los centros educativos ya que los recursos que se utilizan actúan como guía metodológica.

- Formativa: el material que se utiliza en el aprendizaje en ocasiones reincide. Los medios, a más de transmitir contenidos, trasmiten visiones del mundo, es así que, en ocasiones los mensajes son más fáciles de interpretar y otros son indirectos, es decir, difíciles de entender y, por ende, hay que aplicar un pausado análisis de los mismos. Los medios impulsan al análisis crítico y a la reflexión, es por ello que, el docente debe seleccionar cuidadosamente los que se va a utilizar.

Los medios son utilizados como herramienta para incentivar la motivación, ya que cuando más dinámica es la manera de presentar el contenido mayor va a ser la atención del estudiante. Los medios en determinadas ocasiones ayudan a los estudiantes a desarrollar el pensamiento, expresar sus sentimientos, emociones, entre otros. Los medios didácticos permiten al estudiante concretar y aclarar conceptos abstractos.

\subsection{El profesor y el uso de medios y tecnologías}

Con la implementación de las tecnologías en la formación de los futuros profesionales, los docentes deben estar debidamente preparados y capacitados en el manejo y ejecución de las nuevas metodologías para guiar a los estudiantes en el desarrollo de sus actividades. Los docentes no solo deben enfocarse en transmitir conocimientos, sino también en formar alumnos con valores, los mismos que adquieran una personalidad creadora, independiente, capaz de buscar, analizar y estructurar información.

Antonio Bautista (2004) manifiesta que el tipo de uso que el docente da a los medios en sus prácticas, sigue las distinciones entre teorías del currículo: técnica, práctica o interpretativa y crítica. La técnica usa transmisores/reproductores tanto el docente como el estudiante repiten la información que se encuentra en textos, internet, entre otros. Los usos prácticos/situacionales utilizan los medios para llegar a un análisis y comprensión de la información. La crítica permite realizar representaciones, la resolución de problemas, el análisis de información y aprender a utilizar sistemas de representación que son básicos y necesarios para interpretar, entender y relacionarse con el contexto social, físico y cultural (Bautista, 2004, p. 56-57).

Consideramos que el uso crítico de los medios debe ser entendido como la utilización que hacen de los mismos los profesores que asumen la función de intelectuales críticos y transformativos. Este tipo de uso contempla los recursos como herramientas de investigación y, como tal, 
la utilización de los mismos lleva implícita un análisis (Bautista, 2004, p. $52)$.

Con base en análisis propuesto el autor expresa que los medios que se utilizan en el proceso de enseñanza-aprendizaje deben ser los adecuados. El docente debe encontrarse en constante actualización de las nuevas herramientas tecnológica. Los estudios existentes en torno a la utilización de los medios señalan que existe una cierta tendencia a que la utilización de estos gire en torno a dos fines fundamentales: según Cabero la motivación de los alumnos y la transmisión de información. De esta manera se limitan otras posibilidades, por ejemplo, la utilización de los medios para la formación y el perfeccionamiento del docente, la creación y modificación de actitudes, o la evaluación de los estudiantes (Cabero et al., 2003).

\subsection{Formación de los profesores en nuevas tecnologías}

Los ámbitos mencionados anteriormente deben ser considerados como básicos y deben estar en constante revisión y ampliación de conocimientos. Cabe mencionar que la competencia tecnológica nunca dejará de innovar y mostrar avances innovadores virtuales que permitirán una mejor educación.

Bajo los criterios analizados es importante rescatar que las planificaciones de formación de los docentes en aspectos como los medios y nuevas tecnologías, no siempre deben ser realizadas con fundamentación técnica y estética. Se debe poner énfasis en ayudar a los docentes en la integración curricular de medios e instrumentos didácticos utilizados en la mediación pedagógica.

\section{Consideraciones para la incorporación de las TIC en la educación superior}

Menciona Cabero (2003), que la tecnología debe ser cercana y de fácil acceso para los docentes y el alumnado, ya que no es suficiente crear "aulas de informática", sino cambiar el concepto a "la informática al aula", de forma que la tecnología se encuentre a disposición del profesorado cuando desee incorporarla a la práctica de la enseñanza, y sea él exclusivamente, apoyándose en criterios metodológicos, el que decida, o no, su incorporación (Cabero,2003, pág. 45).

En este sentido, así como en épocas de educación tradicional la pizarra era un recurso indispensable en la formación de un individuo, hoy en día los recursos tecnológicos son también indispensables y necesarios en la formación educativa. El profesor, además de mostrar sus virtudes como docente en el aula, debe tener conocimientos en herramientas TIC para un aprendizaje interactivo. La incorporación de las TIC en el ámbito educativo de las universidades tiene un alto nivel de importancia, yaque en ella permitirá ahorrar tiempo y ganar en fiabilidad. La implementación de recursos tecnológicos en el proceso de enseñanza promueve un cambio en instituciones de educación superior que, permitirán formar profesionales capaces de enfrentar la sociedad actual.

\subsection{Alfabetización digital}

Para lograr una correcta y eficiente implementación de recursos tecnológicos el docente debe presentar actitudes y aptitudes favorables para la utilización de las TIC. El Ministerio de Educación, Cultura y Deporte de España (MECD) y la Organización para la Cooperación y el Desarrollo Económicos (OCDE) (2003) se refiere a un sofisticado repertorio de competencias que impregnan el lugar de trabajo, la comunidad y la vida social, entre las que se incluyen las habilidades necesarias para manejar la información y la capacidad de evaluar 
la relevancia y la fiabilidad de lo que busca en Internet (MECD y OCDE, 2003, p. 4). Bajo este análisis, debe ser superado el concepto del simple conocimiento de saber manejar un computador, como tradicionalmente se ha entendido y se sigue entendiendo por ciertos sectores. Para superar estas brechas es necesario formar al profesorado.

\subsection{Formación del profesorado}

Vinculado a esta temática hay una extensa y muy valiosa investigación que data desde la aparición de la educación a distancia. Nos limitaremos a mencionar un extracto de lo comentado por Cabero, Duarte y Barroso (1999-2001) en el que señala:

...voy a remitir al lector interesado, diré aquí solamente que para mí esta
formación debe de superar la visión instrumental que muchas veces
tenemos de la misma, y tiene que adquirir otras dimensiones:
instrumental, semiológica/estética, curricular, pragmática, psicológica,
productora/diseñadora, seleccionadora/evaluadora, crítica,
organizativa, actitudinal e investigadora (p.68).

Con este aporte significativo nos invita a reflexionar que no solo la educación instrumental es necesaria, sino que además es indispensable la educación en valores, paradigma de formación en persona. Esto permitirá armonizar los valores como: el compromiso, responsabilidad, autonomía para incorporar en la planificación, diseño, y evaluación, estrategias que potencien saberes innovadores en formación integral entre profesores y estudiantes.

\subsection{Competencias digitales}

Para lograr desarrollar actividades de aprendizaje y a trabajar con éxito en una sociedad cada vez más exigente e innovadora alineada a saberes actualizados basada en el conocimiento, los estudiantes y docentes deben dominar la tecnología digital con eficacia. Dentro del proceso de enseñanza aprendizaje las TIC pueden aportar en el desarrollo de capacidades como: expertos en la utilización de tecnologías de la información; buscadores, analizadores y evaluadores de información; solucionadores de problemas; creativos e innovadores de herramientas de productividad; comunicadores, colaboradores y, lo más importante, ciudadanos informados, responsables y capaces de contribuir a la sociedad.

En la actualidad, un docente en ejercicio necesita estar capacitado para poder ofrecer a sus estudiantes oportunidades de aprendizaje que se apoyen en las TIC. Debe tener conocimientos de cómo utilizarlas y saber cómo éstas pueden contribuir al aprendizaje de los estudiantes. Para desarrollar capacidades en los estudiantes es necesario que el currículo de educación actual fortalezcalas competencias profesionales de un docente. Los docentes necesitan estar formados para enseñar a los estudiantes las ventajas que aporta el uso de las TIC, de modo que se integren este tipo de recursos de forma eficaz en las asignaturas. De acuerdo a lo señalado por la UNESCO (2008) respecto a las competencias TIC se determinan a criterio personal las siguientes competencias:

- Conocer el funcionamiento básico del hardware y del software, así como las aplicaciones de productividad, un navegador de Internet, un programa de comunicación, un presentador multimedia y aplicaciones de gestión.

- Conocer una variedad de aplicaciones y herramientas específicas, las cuales deben ser capaces de utilizarlas con flexibilidad en diferentes situaciones basadas en problemas y proyectos. Los docentes deben poder utilizar redes de recursos para ayudar a los estudiantes a colaborar, acceder a la información y comunicarse con expertos externos, a fin de analizar y resolver los problemas seleccionados. Los 
docentes también deberán estar en capacidad de utilizar las TIC para crear y supervisar proyectos de clase realizados individualmente o por grupos de estudiantes.

- Tener la capacidad de diseñar comunidades de conocimiento basadas en las TIC, y también saber utilizar estas tecnologías para apoyar el desarrollo de las habilidades de los estudiantes, tanto en materia de creación de conocimientos como para su aprendizaje permanente y reflexivo.

\subsection{Competencias de los docentes de sigloXXI}

Sobre las competencias de los docentes del siglo XXI García Vallinas (2007) afirma:

Las competencias necesarias para desenvolverse en el amplio, plural, dinámico e incierto mercado de trabajo se valora precisamente por la flexibilidad o capacidad de adaptación del trabajador o trabajadora a entornos laborales diversos; pero también, más allá de las demandas cambiantes del mercado de trabajo, importan las necesidades $\mathrm{y}$ requerimientos formativos de las personas, trascender la formación específica o especializada para determinados empleos, dotándoles de una formación más general que atienda a su realización personal como ciudadanos y ciudadanas o les capacite para decidir con criterio el rumbo y el sentido que atribuirán a sus propias vidas (p.1).

Por lo expuesto, se puede determinar que un docente regido a los cambios de la sociedad actual debe implementar los diversos recursos tecnológicos al momento de impartir sus conocimientos e incentivar a sus estudiantes a practicar el auto aprendizaje. Desarrollar en ellos capacidades como el análisis, la reflexión y la comprensión de información requiere formar competencias. Al respecto, Gonzales (2012) indica algunas de las capacidades que deben ser desarrolladas en la educación actual:

- Crear y editar videos como recursos educativos para la enseñanza;

- Usar blogs y wikis para generar plataformas de aprendizaje en línea dirigidas a sus estudiantes;

- Aprovechar las imágenes web para su uso en el aula;

- Utilizar las redes sociales dentro del proceso enseñanza aprendizaje como un medio para conectarse con colegas y estudiantes que les permitan crecer profesionalmente;

- Compilar e-portafolios para su autodesarrollo;

- Tener un conocimiento profundo de los tipos de seguridades online, utilización de software o aplicaciones que permitan detectar el plagio en los trabajos de sus estudiantes;

- Crear videos con capturas de pantalla y vídeo-tutoriales;

- Recopilar contenido web apto para el aprendizaje en el aula;

- Usar y proporcionar a los estudiantes las herramientas de gestión de tareas necesarias para organizar su trabajo y planificar su aprendizaje de forma óptima;

- Entender las cuestiones relacionadas con derechos de autor y uso honesto de los materiales;

- Utilizar herramientas digitales para crear cuestionarios de evaluación;

- Usar herramientas de colaboración para la construcción y edición de textos;

- Encontrar y evaluar el contenido web;

- Usar dispositivos móviles (tablets o smartphones);

- Identificar recursos didácticos online seguros para los estudiantes; 
- Conocer el uso de YouTubey sus potencialidades dentro del aula, usar herramientas de anotación y compartir ese contenido con sus alumnas y alumnos;

- Compartir las páginas web y las fuentes de los recursos que ha expuesto en clase;

- Usar organizadores gráficos, online e imprimibles;

- Usar herramientas para crear y compartir tutoriales con la grabación fílmica de capturas de pantalla;

- Aprovechar las herramientas de trabajo online en grupo o en equipo que utilizan mensajería;

- Buscar eficazmente en Internet empleando el mínimo tiempo posible;

- Llevar a cabo un trabajo de investigación utilizando herramientas digitales;

- Usar herramientas para compartir archivos y documentos con los estudiantes.

Sin embargo, es importante referirse a algunas competencias claves como: manejar y saber enseñar el uso de plantillas y hojas de cálculo; conocer y enseñar métodos de investigación digital, como las competencias en el manejo de información-CMI. Además, es necesario conocer y manejar herramientas de edición gráfica digital; reflexionar y enseñar a sus estudiantes los usos prácticos, críticos y éticos de la red; conocer y gestionar aulas virtuales; usar foros digitales con los estudiantes. Actualmente, es una necesidad el conocimiento de las TIC, pues, todo profesional requiere desarrollar efectividad en su profesión. A continuación, se detalla el análisis sobre la utilización de las TIC en la Universidad Central del Ecuador.

Los cuestionarios que se presentan muestran el número de participantes. El cuestionario fue contestado de manera voluntaria por 300 docentes en el periodo 2015-2016 y de igual forma por 1.799 estudiantes.

\begin{tabular}{|c|c|c|c|c|}
\hline Género & Frecuencia & Porcentaje & $\begin{array}{l}\text { Porcentaje } \\
\text { Válido }\end{array}$ & $\begin{array}{l}\text { Porcentaje } \\
\text { Acumulado }\end{array}$ \\
\hline Masculino & 204 & $\overline{68,0}$ & 123 & 68,0 \\
\hline Femenino & 96 & 32,0 & 32,0 & 100 \\
\hline Total & 300 & 100,0 & 100,0 & \\
\hline
\end{tabular}

Cuadro 1. Frecuencia y porcentajes en función del género de los docentes de la UCE

\begin{tabular}{|c|c|c|c|c|}
\hline Género & Frecuencia & Porcentaje & $\begin{array}{l}\text { Porcentaje } \\
\text { Válido }\end{array}$ & $\begin{array}{l}\text { Porcentaje } \\
\text { Acumulado }\end{array}$ \\
\hline Masculino & 824 & 45,8 & 45,9 & 45,9 \\
\hline Femenino & 970 & 53,9 & 54,1 & 100,0 \\
\hline Total & 1794 & 99,7 & 100,0 & \\
\hline Perdidos & 5 & 0,3 & & \\
\hline Total & 1799 & 100 & & \\
\hline
\end{tabular}

Cuadro 2. Frecuencia y porcentajes en función del género de los estudiantes de la UCE

\section{INSTRUMENTO Y ANÁLISIS DE DATOS}

El cuestionario habilidades instrumentales en el uso de las TIC (docentes) fue elaborado basándose en las competencias y estándares TIC de la UNESCO, el mismo que fue validado por el Panel Internacional de Investigación en Tecnología Educativa-PI2TE mencionados en el marco conceptual, y que se agrupan en 3 dimensiones. La primera dimensión tiene que ver con habilidades instrumentales en el uso de las TIC, mismo que tiene 9 ítems; la segunda dimensión tiene que ver con habilidades didáctico-metodológicas en el uso de las TIC, que 
a su vez está conformada por 11 ítems y, por último, la dimensión importancia del uso de las TIC en la práctica docente, tales dimensiones comprenden a su vez 11 ítems. Las 3 dimensiones de este primer instrumento han sido desglosadas en un total de 31 ítems que configuran el presente cuestionario, y que son contestados mediante una escala continua de cinco puntos según la escala de Likert, desde: Totalmente de acuerdo, De acuerdo (2), Indeciso/a (3), Desacuerdo (4) y Totalmente en desacuerdo (5) con una muestra de estudiantes (alpha $=0.85$ ). Los datos fueron organizados, codificados $\mathrm{y}$ analizados utilizando el paquete estadístico SPSS versión 22.0.

La encuesta sobre plataforma educativa-web 2.0 para docentes y estudiantes, fue elaborado basándose en la percepción de uso de la plataforma sakai que funciona en la UCE el mismo que fue validado por PI2TE mencionado en el marco conceptual, y que se encuentra compuesto por 22 preguntas que están conformadas por las dimensiones de género, demografía, valoración de la plataforma virtual, uso de herramientas de la web 2.0 y redes. Los datos fueron organizados, codificados y analizados utilizando el paquete estadístico SPSS versión 22.0. 


\section{PARTE 1}

\begin{tabular}{|c|c|c|c|c|c|c|c|c|c|c|c|c|}
\hline \multicolumn{4}{|c|}{ SOFTWARE EDUCATIVO (APRENDIZAJE) } & \multicolumn{3}{|c|}{$\begin{array}{l}\text { FUENTE DE INFORMACIÓN } \\
\text { (ACTUALIZACIÓN DE } \\
\text { CONOCIMIENTOS) }\end{array}$} & \multicolumn{3}{|c|}{$\begin{array}{l}\text { INDICADOR ESPACIOS } \\
\text { VIRTUALES (FORMACION } \\
\text { DOCENTES) }\end{array}$} & \multicolumn{3}{|c|}{ TOTAL } \\
\hline DATOS & $\begin{array}{l}\text { Informante } \\
\text { s } \\
\text { C }\end{array}$ & $\begin{array}{l}\% \text { del } \\
\text { total IC }\end{array}$ & $\begin{array}{l}\% \\
\text { ACUMULADO } \\
\text { IC }\end{array}$ & $\begin{array}{l}\text { informantes } \\
\text { C }\end{array}$ & $\begin{array}{c}\% \text { del } \\
\text { total IC }\end{array}$ & $\begin{array}{l}\% \\
\text { CUMULADO } \\
\text { C }\end{array}$ & $\begin{array}{l}\text { informant } \\
\text { s C }\end{array}$ & $\begin{array}{l}\% \text { del } \\
\text { total } \\
\text { IC }\end{array}$ & $\begin{array}{l}\% \\
\text { ACUMULAD } \\
\text { O IC }\end{array}$ & $\begin{array}{l}\text { informante } \\
\text { s C }\end{array}$ & $\begin{array}{l}\% \text { del } \\
\text { total IC }\end{array}$ & $\begin{array}{l}\% \\
\text { ACUMULAD } \\
\text { O IC }\end{array}$ \\
\hline Totalmente & 104 & $34,78 \%$ & $34,78 \%$ & 85 & $28,50 \%$ & $28,50 \%$ & 70 & $\begin{array}{r}23,24 \\
\%\end{array}$ & $23,24 \%$ & 86 & $28,84 \%$ & $28,84 \%$ \\
\hline $\begin{array}{l}\text { En gran } \\
\text { medida }\end{array}$ & 96 & $32,11 \%$ & $66,89 \%$ & 103 & $34,61 \%$ & $63,11 \%$ & 87 & $\begin{array}{r}29,10 \\
\%\end{array}$ & $52,34 \%$ & 95 & $31,94 \%$ & $60,78 \%$ \\
\hline $\begin{array}{l}\text { En regular } \\
\text { medida }\end{array}$ & 68 & $22,58 \%$ & $89,46 \%$ & 72 & $24,21 \%$ & $87,32 \%$ & 87 & $\begin{array}{r}29,10 \\
\%\end{array}$ & $81,44 \%$ & 76 & $25,29 \%$ & $86,08 \%$ \\
\hline $\begin{array}{l}\text { En baja } \\
\text { medida }\end{array}$ & 26 & $8,70 \%$ & $98,16 \%$ & 32 & $10,80 \%$ & $98,12 \%$ & 48 & $\begin{array}{r}15,89 \\
\%\end{array}$ & $97,32 \%$ & 35 & $11,79 \%$ & $97,87 \%$ \\
\hline $\begin{array}{l}\text { En ninguna } \\
\text { medida }\end{array}$ & 6 & $1,84 \%$ & $100,00 \%$ & 6 & $1,88 \%$ & $100,00 \%$ & 8 & $2,68 \%$ & $100,00 \%$ & 6 & $2,13 \%$ & $100,00 \%$ \\
\hline TOTAL & 299 & $100,00 \%$ & & 299 & $\begin{array}{r}100,00 \\
\%\end{array}$ & & 299 & $\begin{array}{r}100,0 \\
0 \%\end{array}$ & & 299 & $\begin{array}{r}100,00 \\
\%\end{array}$ & \\
\hline
\end{tabular}

Cuadro 3. Factores análisis univariante habilidades instrumentales en el uso de las TIC (docentes) 
De la información obtenida de 299 informantes claves que representa el 66,89\% acumulado entre los indicadores "totalmente" y "en gran medida" con respecto al uso de software para promover el aprendizaje en procesos de enseñanza aprendizaje, y con base en una encuesta realizada en Argentina, Chile, Costa Rica y México se mostró que más del $60 \%$ de los docentes usa poco o nada las herramientas tecnológicas (Informe en Tendencias Sociales Educativas, 2014, p. 180).

Según los datos expuestos se puede inferir que el diseño de estrategias para eliminar barreras, incluye la incorporación de las TIC al currículo, ya que el uso de software educativo nos permite promover aprendizajes significativos. El uso de las TIC motiva al estudiante a crear y generar nuevo conocimiento. Bajo esta premisa y en función de los criterios expresados por los docentes respecto al uso de software educativo para promover aprendizajes con sus estudiantes, podemos inferir que es totalmente importante. Su uso permitirá reforzar lo aprendido dentro del aula de una manera interactiva.

De la información obtenida de 188 informantes claves que representa el 63,11\% acumulado entre los indicadores "totalmente" y en "gran medida" con respecto a la fuente de información actualización de conocimientos, y de acuerdo al informe en Tendencias Sociales Educativas de 2014, la actividad que más realizan en sus computadoras los docentes para la gestión escolar consiste en escribir documentos o comunicados (98\%). Buscar información (98\%). Preparar o crear presentaciones (95\%). Hacer cronogramas u horarios (93\%), mientras que la actividad menos realizada es comunicarse con sus alumnos (46\%), tomando en cuenta estos datos a criterio personal se considera importante que el docente utilice las TICpara: reforzar lo aprendido en el aula mediante el uso de revistas digitales, ebooks, blogs, entre otras herramientas; para buscar fuentes confiables de información. Todos estos elementos permiten que los docentes tengan acceso a información científica y adquieran competencias que les permitan navegar de forma eficiente y segura en el internet.

De la información obtenida de 157 informantes claves que representan el 75,58\% acumulado entre los indicadores "totalmente" y en "gran medida" con respecto a la fuente de información formación docentes y tomando en cuenta el informe de la Comisión Económica para América Latina y el Caribe (CEPAL) se muestra que el porcentaje de docentes que ha tomado cursos sobre TIC es bastante alto en países como Chile (90\%), Perú (82\%), Colombia (61\%), Costa Rica (60\%), mientras el porcentaje de docentes que ha tomado cursos sobre TIC es bastante bajo en países en vías de desarrollo como Nicaragua $(17 \%)$, Paraguay $(10 \%)$ o Guatemala (6\%). Se puede inferir que la actualización docente como parte fundamental en el desarrollo educativo docente-estudiante, permite al docente publicar y compartir información en la web. El objetivo es trabajar de manera colectiva con otros docentes y así poder unificar conocimientos con el objetivo de aumentar la base de inteligencia general para una mejor formación docente. El cuadro cuatro que se muestra a continuación, muestra el nivel de importancia que los estudiantes le dan al uso de la plataforma virtual.

Licencia Creative Commons Atribución Internacional 4.0 (CC BY 4.0)

Revista Cátedra, 2(1), pp. 76-97, enero-abril 2019. e-ISSN: 2631-2875

https://doi.org/10.29166/catedra.v2i1.1560 


\begin{tabular}{|c|c|c|c|c|c|c|c|c|c|c|c|c|c|c|c|c|c|c|}
\hline \multicolumn{4}{|c|}{$\begin{array}{l}\text { IMPORTANCIA DEL USO DE LA } \\
\text { PLATAFORMA }\end{array}$} & \multicolumn{3}{|c|}{$\begin{array}{l}\text { VALORACIÓN DE LA } \\
\text { PLATAFORMA }\end{array}$} & \multicolumn{3}{|c|}{$\begin{array}{l}\text { USO Y APRENDIZAJE DE } \\
\text { PLATAFORMA } \\
\text { VIRTUALES }\end{array}$} & \multicolumn{3}{|c|}{ ETICIDAD } & \multicolumn{3}{|c|}{ USO DE LA WEB 2.0} & \multicolumn{3}{|c|}{ TOTAL, ENCUESTAS } \\
\hline Datos & $\begin{array}{l}\text { inf } \\
\text { or } \\
\text { ma } \\
\text { nte } \\
\text { s C }\end{array}$ & $\begin{array}{l}\% \text { del } \\
\text { total IC }\end{array}$ & $\begin{array}{l}\% \\
\text { ACUMU } \\
\text { LADO IC }\end{array}$ & $\begin{array}{l}\text { inform } \\
\text { antes } \\
\text { C }\end{array}$ & $\begin{array}{l}\text { \% del } \\
\text { total } \\
\text { IC }\end{array}$ & $\begin{array}{l}\text { \% } \\
\text { ACUMU } \\
\text { LADO } \\
\text { IC }\end{array}$ & $\begin{array}{l}\text { informa } \\
\text { ntes } C\end{array}$ & $\begin{array}{l}\text { \% del } \\
\text { total } \\
\text { IC }\end{array}$ & $\begin{array}{l}\% \\
\text { ACUMU } \\
\text { LADO IC }\end{array}$ & $\begin{array}{l}\text { infor } \\
\text { mante } \\
\text { s C }\end{array}$ & $\begin{array}{l}\text { \% del } \\
\text { total IC }\end{array}$ & $\begin{array}{l}\% \\
\text { ACUMUL } \\
\text { ADO IC }\end{array}$ & $\begin{array}{l}\text { inf } \\
\text { or } \\
\text { ma } \\
\text { nt } \\
\text { es } \\
\text { C }\end{array}$ & $\begin{array}{l}\text { \% del } \\
\text { total } \\
\text { IC }\end{array}$ & $\begin{array}{l}\% \\
\text { ACUM } \\
\text { ULAD } \\
\text { O IC }\end{array}$ & $\begin{array}{l}\text { infor } \\
\text { mant } \\
\text { es C }\end{array}$ & $\begin{array}{l}\text { \% del } \\
\text { total } \\
\text { IC }\end{array}$ & $\begin{array}{l}\% \\
\text { ACU } \\
\text { MUL } \\
\text { ADO } \\
\text { IC }\end{array}$ \\
\hline NUNCA & 87 & $4,82 \%$ & $4,82 \%$ & 139 & $7,73 \%$ & $7,73 \%$ & 281 & $\begin{array}{r}15,60 \\
\%\end{array}$ & $15,60 \%$ & 45 & $2,50 \%$ & $2,50 \%$ & 45 & $2,50 \%$ & $2,50 \%$ & 119 & $6,63 \%$ & $\begin{array}{r}6,63 \\
\%\end{array}$ \\
\hline $\begin{array}{l}\text { MUY } \\
\text { POCAS } \\
\text { VECES }\end{array}$ & $\begin{array}{r}17 \\
3\end{array}$ & $9,59 \%$ & $14,41 \%$ & 336 & $\begin{array}{r}18,69 \\
\%\end{array}$ & $\begin{array}{r}26,42 \\
\%\end{array}$ & 181 & $\begin{array}{r}10,04 \\
\%\end{array}$ & $25,63 \%$ & 108 & $5,99 \%$ & $8,49 \%$ & $\begin{array}{r}10 \\
8\end{array}$ & $5,99 \%$ & $8,49 \%$ & 181 & $\begin{array}{r}10,06 \\
\%\end{array}$ & $\begin{array}{r}16,6 \\
9 \%\end{array}$ \\
\hline $\begin{array}{l}\text { ALGUNAS } \\
\text { VECES }\end{array}$ & $\begin{array}{r}39 \\
0\end{array}$ & $21,66 \%$ & $36,08 \%$ & 551 & $\begin{array}{r}30,65 \\
\%\end{array}$ & $\begin{array}{r}57,07 \\
\%\end{array}$ & 271 & $\begin{array}{r}15,05 \\
\%\end{array}$ & $40,68 \%$ & 289 & $\begin{array}{r}16,04 \\
\%\end{array}$ & $24,53 \%$ & $\begin{array}{r}28 \\
9\end{array}$ & $\begin{array}{r}16,04 \\
\%\end{array}$ & $\begin{array}{r}24,53 \\
\%\end{array}$ & 358 & $\begin{array}{r}19,89 \\
\%\end{array}$ & $\begin{array}{r}36,5 \\
8 \%\end{array}$ \\
\hline $\begin{array}{l}\text { CASI } \\
\text { SIEMPRE }\end{array}$ & $\begin{array}{r}62 \\
9\end{array}$ & $34,95 \%$ & $71,03 \%$ & 475 & $\begin{array}{r}26,41 \\
\%\end{array}$ & $\begin{array}{r}83,48 \\
\%\end{array}$ & 210 & $\begin{array}{r}11,70 \\
\%\end{array}$ & $52,38 \%$ & 475 & $\begin{array}{r}26,39 \\
\%\end{array}$ & $50,91 \%$ & $\begin{array}{r}47 \\
5\end{array}$ & $\begin{array}{r}26,39 \\
\%\end{array}$ & $\begin{array}{r}50,91 \\
\%\end{array}$ & 453 & $\begin{array}{r}25,17 \\
\%\end{array}$ & $\begin{array}{r}61,7 \\
4 \%\end{array}$ \\
\hline SIEMPRE & $\begin{array}{r}49 \\
3\end{array}$ & $27,39 \%$ & $98,42 \%$ & 183 & $\begin{array}{r}10,19 \\
\%\end{array}$ & $\begin{array}{r}93,67 \\
\%\end{array}$ & 184 & $\begin{array}{r}10,23 \\
\%\end{array}$ & $62,60 \%$ & 737 & $\begin{array}{r}40,97 \\
\%\end{array}$ & $91,88 \%$ & $\begin{array}{r}73 \\
7\end{array}$ & $\begin{array}{r}40,97 \\
\%\end{array}$ & $\begin{array}{r}91,88 \\
\%\end{array}$ & 467 & $\begin{array}{r}25,95 \\
\%\end{array}$ & $\begin{array}{r}87,6 \\
9 \%\end{array}$ \\
\hline 9 & 29 & $1,58 \%$ & $\begin{array}{r}100,00 \\
\%\end{array}$ & 114 & $6,33 \%$ & $\begin{array}{r}100,0 \\
0 \%\end{array}$ & 673 & $\begin{array}{r}37,40 \\
\%\end{array}$ & $\begin{array}{r}100,00 \\
\%\end{array}$ & 146 & $8,12 \%$ & $\begin{array}{r}100,00 \\
\%\end{array}$ & $\begin{array}{r}14 \\
6\end{array}$ & $8,12 \%$ & $\begin{array}{r}100,0 \\
0 \%\end{array}$ & 221 & $\begin{array}{r}12,31 \\
\%\end{array}$ & $\begin{array}{l}100, \\
00 \%\end{array}$ \\
\hline TOTAL & $\begin{array}{l}17 \\
99\end{array}$ & $\begin{array}{r}100,00 \\
\% \\
\end{array}$ & & 1799 & $\begin{array}{r}100,0 \\
0 \% \\
\end{array}$ & & 1799 & $\begin{array}{r}100,0 \\
0 \%\end{array}$ & & 1799 & $\begin{array}{r}100,00 \\
\% \\
\end{array}$ & & $\begin{array}{l}17 \\
99\end{array}$ & $\begin{array}{r}100,0 \\
0 \% \\
\end{array}$ & & $\begin{array}{r}179 \\
9 \\
\end{array}$ & $\begin{array}{r}100,0 \\
0 \% \\
\end{array}$ & \\
\hline
\end{tabular}

Cuadro 4. Factores análisis univariante plataforma educativa-web 2.0 UCE (estudiantes) 
Con base en los resultados obtenidos de 1.122 informantes claves que representa el 62,34\% acumulado entre los indicadores "siempre" y "casi siempre" con respecto al indicador importancia del uso de la plataforma educativa en la UCE y de acuerdo a los datos obtenidos en la Revista de Tecnología Journal Technology (2013) se menciona que a nivel latinoamericano el $68.5 \%$ de los estudiantes consideran importante el uso de la plataforma virtual. Las relaciones entre los estudiantes y los docentes incrementan y contribuyen en el desarrollo de las actividades y permiten apreciar el rendimiento académico durante el desarrollo del curso. De acuerdo a esta información se puede observar que el indicador importancia del uso de la plataforma educativa en la UCE en los estudiantes, se encuentra a la par en relación con otras universidades, pero es necesario incentivar a los estudiantes a utilizar la plataforma virtual de aprendizaje como instrumento de estudio en todos los escenarios educativos.

De igual forma de los 658 informantes claves que representa el $36,60 \%$ acumulado entre los indicadores "siempre" y "casi siempre" con respecto al indicador valoración de la plataforma educativa y de acuerdo a estudios realizados por la Asociación para el Desarrollo de la Tecnología Educativa y de las Nuevas Tecnologías Aplicadas a la Educación-EDUTEC (2013) en su artículo "Uso de los entornos virtuales de aprendizaje en la educación superior", en referencia a la valoración de los Entornos Virtuales de Aprendizaje-EVA, el 100\% de las universidades consultadas expresó que los consideran muy adecuados. Esto permite ofrecer la oportunidad de incorporar elementos conceptuales, procedimentales y actitudinales que facilitan una formación en competencias en los estudiantes y afianzamiento de la interculturalidad al trascender las fronteras impuestas por la distancia. De acuerdo a estos datos es necesario incentivar la utilización de la plataforma virtual de aprendizaje de la UCE, motivando al estudiante mediante la utilización de diversas actividades que forman parte de la plataforma y que permitan fortalecer el aprendizaje y utilización de las herramientas que conforman la misma.

En lo que se refiere a resultados de otras competencias de los 394 informantes claves que representa el 21,93\% acumulado entre los indicadores "siempre" y "casi siempre" con respecto al indicador uso y aprendizaje de plataformas virtuales y de acuerdo al artículo "Uso de los entornos virtuales de aprendizaje en la educación superior" de EDUTEC (2013), el 60\% de los estudiantes respondió que utilizan la plataforma en actividades de aprendizaje. Las actividades que generalmente se usa son: materiales de apoyo y entrega de deberes. De esto se puede inferir que es necesario que docentes y estudiantes aprendan a usar todas las herramientas que brinda la plataforma virtual.

Asimismo, de los 1.212 informantes claves que representa el 67,36\% acumulado entre los indicadores "siempre" y "casi siempre" con respecto al indicador eticidad y según un artículo de Technology and Ethics (2002) muestra un aumento de disponibilidad de material ofensivo y peligroso en Internet en un 76\%. Monopolios en la industria del software y de la información en un $60 \%$, referido a lo anterior resulta claro que la globalización vinculada a Internet parecería requerir de normas de carácter también global. El objetivo sería establecer patrones de comportamiento, es decir, una defensa de valores humanos.

En referencia a otros datos obtenidos, se analiza que de 1.212 informantes claves que representa el 67,36\% acumulado entre los indicadores "siempre" y "casi siempre" con respecto al indicador uso de la web 2.0 y de acuerdo a datos de la Universidad Nacional de Educación a Distancia, Sociedad del Conocimiento y Educación, (2012) donde se menciona que el $92,6 \%$ suele acceder, participar o visitar la web 2.0 todos los días. Es necesario implementar gran cantidad de herramientas que ofrece la web 2.0 en el ámbito educativo que permita generar y compartir aprendizajes colaborativos y autónomos, para que se incentive el uso adecuado de estas herramientas tecnológicas.

Licencia Creative Commons Atribución Internacional 4.0 (CC BY 4.0)

Revista Cátedra, 2(1), pp. 76-97, enero-abril 2019. e-ISSN: 2631-2875

https://doi.org/10.29166/catedra.v2i1.1560 


\section{Discusión}

De manera general, los futuros profesionales tienen pleno conocimiento con respecto a su propio nivel de competencia digital y creen ser idóneos para ejercer de manera objetiva dicha competencia. Se tendrá en cuenta como se ha determinado anteriormente en el cuadro No. 3, que la variable competencias TIC docentes queda determinada para la presente investigación a partir del conocimiento de actividades TIC hacia el uso en general en procesos de enseñanza aprendizaje. Para esto se han determinado nueve cuestiones de investigación relacionadas con el conocer, aplicar y valorar (actualización de conocimientos, formación docente, gestión áulica, apoyo al aprendizaje, procesos de evaluación, eticidad, propiedad intelectual y pedagógico didáctico) la tecnología en procesos de enseñanza aprendizaje en el aula y fuera de ella. Así podemos contestar las siguientes preguntas:

- ¿Cuál es el nivel de uso de software educativo de los docentes de la Universidad Central del Ecuador?

Al analizar los datos podemos determinar que los docentes de la UCE presentan porcentajes de conocimiento medianamente altos (66,89\%) con respecto al uso de software educativo para promover el aprendizaje en procesos de enseñanza aprendizaje. El uso del software educativo nos permite promover aprendizajes en los estudiantes ayudándolos en la evaluación, análisis y síntesis de la información; de esta forma se motiva al estudiante a crear y generar nuevos conocimientos.

- ¿Cuál es el grado de actualización de conocimientos respecto a la tecnología que tienen los docentes de la Universidad Central del Ecuador con relación a la gestión áulica a través de la búsqueda de información?

Según los datos obtenidos se analiza que los docentes de la UCE presentan porcentajes de conocimiento medianamente altos $(63,11 \%)$ con respecto a la fuente de información actualización de conocimientos.

- ¿Cuál es el nivel de formación de los docentes de la Universidad Central del Ecuador con respecto a la capacitación en tecnología?

$\mathrm{Al}$ análisis de los datos podemos determinar que los docentes de la UCE presentan porcentajes de conocimiento medianamente altos $(75,58 \%)$ con respecto a la fuente de información formación docentes, y tomando en cuenta el informe de la Comisión Económica para América Latina y el Caribe (CEPAL) se muestra que el porcentaje de docentes que ha tomado cursos sobre TIC es bastante alto en países como: Chile (90\%), Perú (82\%), Colombia (61\%) o Costa Rica (60\%), mientras el porcentaje de docentes que ha tomado cursos sobre TIC es bastante bajo en países en vías de desarrollo como: Nicaragua (17\%), Paraguay (10\%) o Guatemala (6\%).

- ¿Cuál es el grado de aplicación de estrategias metodológicas que aplican los docentes de la Universidad Central del Ecuador para mejorar los procesos de gestión áulica?

$\mathrm{Al}$ análisis de los datos podemos determinar que los docentes de la UCE presentan porcentajes de conocimiento medianamente altos $(43,10 \%)$, con respecto a procesos áulicos.

- ¿Cuál es el grado de apoyo al aprendizaje con tecnologías para fortalecer el trabajo autónomo de los estudiantes?

Al análisis de los datos podemos inferir que los docentes de la UCE presentan porcentajes de conocimiento medianamente altos $(47,77 \%)$ con respecto a la elaboración de recursos multimedios apoyo al aprendizaje y de acuerdo con datos de un estudio en el contexto español, Vaillant y Marcelo (2012) señalan que el 28,5\% de los docentes usan las TIC y el 30\% hace un uso ocasional (menos de una vez al mes). El 41,5\% restante de los profesores manifiesta que hace un uso regular y sistemático de las TIC en sus aulas, aunque con grados de intensidad muy diferentes.

Licencia Creative Commons Atribución Internacional 4.0 (CC BY 4.0)

Revista Cátedra, 2(1), pp. 76-97, enero-abril 2019. e-ISSN: 2631-2875

https://doi.org/10.29166/catedra.v2i1.1560 
Cuando los docentes hacen uso de las tecnologías en su enseñanza, lo hacen para transmitir contenidos como apoyo a la exposición oral (78,7\%), para presentar contenido mediante un sistema multimedia o hipermedia (62,3\%), y para realizar demostraciones que permitan simular determinados escenarios $(44,5 \%)$, y de acuerdo con estos datos se puede interpretar que el uso de recursos multimedia en la educación no está siendo aprovechada en su gran mayoría por los docentes. Solo lo utilizan como apoyo para exposición oral y para transmitir contenidos, olvidando que estas herramientas deben ser utilizadas de forma innovadora en sus clases para generar conocimiento.

- ¿Cómo es el proceso de evaluación que aplican los docentes de la Universidad Central del Ecuador?

Al análisis que reportan los datos podemos determinar que los docentes de la UCE presentan porcentajes de aplicación medianamente $(46,46 \%)$ respecto al indicador de procesos de evaluación, según los estándares de competencia en TIC para docentes de la UNESCO, las herramientas TIC deben servir como herramienta de evaluación. Además, Ecuador es uno de los países donde se utilizan las TIC como herramienta de evaluación, un ejemplo de ello es la realización de las pruebas Ser Bachiller donde participaron 231.759 estudiantes de tercer año de Bachillerato para medir el grado de dominio en los estándares de aprendizaje establecidos por el Ministerio de Educación.

- ¿Qué actitud tienen los docentes hacia la aplicación de la ética en general con respecto al uso de la tecnología en la educación basadas en TIC?

Respecto a la actitud mostrada por los docentes de la UCE hacia la aplicación de la ética en el uso en general de las TIC en la educación, del análisis que reportan los datos podemos determinar que los docentes presentan porcentajes de aplicación muy altos $(83,17 \%)$ con respecto al indicador de eticidad, así mismo, según un artículo de Technology and Ethics. Privacy in the Workplace en Perspectives in Business Ethics (2002) muestra un aumento de disponibilidad de material ofensivo y peligroso en Internet en un 76\%, monopolios en la industria del software y de la información en un $60 \%$. A lo anterior resulta claro que la globalización vinculada a Internet parecería requerir de normas de carácter también global que establezcan patrones de comportamiento y conlleven una defensa global de valores humanos. Esta es por cierto una tarea complicada pues requeriría de leyes de carácter también global que muchos países y gobiernos no parecerían aún estar en disposición de aceptar fácilmente. Es importante rescatar que los docentes consideren en cada una de sus tareas las evidencias sobre la importancia de la ética en todos los ámbitos de la vida.

- ¿Qué actitud tienen los docentes hacia el respeto por la propiedad intelectual cuando se usa tecnología en procesos educativos?

Respecto a la actitud mostrada por los docentes de la Universidad Central del Ecuador hacia el respeto por la propiedad intelectual en el uso de tecnología en educación el análisis que reportan los datos $(87,55 \%)$ es muy alto con respecto al indicador de propiedad intelectual. De acuerdo con estudios realizados por McCabe a 2.294 estudiantes de secundaria de 25 escuelas públicas y privadas de EE.UU., se obtuvo que un $52 \%$ de estudiantes había copiado párrafos explícitos desde algún sitio web sin realizar la correspondiente cita. Además, cabe destacar una investigación sudamericana realizada por Bordigon et al., (2011) la cual señaló que 
"el 50\% de los alumnos de educación básica y media del segmento escolar argentino declaran haber copiado para confeccionar sus tareas y trabajos" (p. 34). En vista de los datos obtenidos en la encuesta y el alto índice de plagio realizado por los estudiantes, es de vital importancia que los centros de educación superior tomen medidas para prevenir y reducir el fraude académico mediante la implementación y utilización de programas virtuales que detecten antiplagio por parte de los docentes y así garantizar una cultura de respeto a la autoría (honestidad académica) y potenciar el manejo de información confiable.

- ¿Qué actitud tienen los docentes con respecto al apoyo de las tecnologías en procesos pedagógicodidácticos?

Al análisis de los datos podemos inferir que los docentes de la Universidad Central del Ecuador presentan porcentajes de conocimiento altos $(87,23 \%)$ con respecto al indicador pedagógico didáctico. Además, según estudios de Vaillant y Marcelo (2012) en el contexto español, la mayor parte de docentes (78,7\%) hacen uso de tecnologías en su enseñanza para transmitir contenidos como apoyo a la exposición oral, olvidando los aspectos pedagógicos que deben contemplar el uso de las tecnologías, con base en lo referido. Es necesario que se dé una eficiente apropiación y gestión de las TIC a la luz de la nueva visión de los procesos de aprendizaje, que contribuya a orientar las políticas educativas, la organización de la institución, los recursos materiales y los actores involucrados. No se trata de hacer lo mismo de otra manera, sino de modificar los propios objetivos en función de los requerimientos que plantea el uso de las tecnologías para articular la práctica pedagógica con los procesos y productos tecnológicos.

\section{Conclusiones}

Las experiencias sistematizadas con base en la presentación de datos y los respectivos análisis ven a las tecnologías un paradigma innovador que rápidamente se inserta en la educación superior del siglo XXI. Para concluir con la investigación se deduce que al existir cambios y ritmos vertiginosos pedagógicos modernos, se reflexiona desde dos ámbitos: el primero se relaciona a las competencias que los docentes han desarrollado en los procesos de enseñanza-aprendizaje, por lo que es claro inferir que se puede mejorar o desarrollar algunas habilidades instrumentales en los docentes para que faciliten a los estudiantes mejores herramientas tecnológicas que ofrece la web 2.0, cuyo propósito es integrar abordajes sistémicos que integre la teoría y la práctica relacionados a contextos profesionales.

El siguiente ámbito se relaciona a la percepción de los futuros profesionales de la Universidad Central del Ecuador, en la que se demuestra que, si bien los hombres usan más tecnología, las mujeres no se encuentran con valores significativamente excelentes como para poder establecer que el género masculino ha desarrollado más competencias que el femenino.

En fin, la investigación realizada centra la atención en los docentes y estudiantes de la Universidad Central del Ecuador, pero es notorio según el análisis que en los docentes el uso de las herramientas de la web 2.0 causa dificultad en la mediación pedagógica en escenarios educativos principalmente en la incorporación de herramientas tecnológicas para potenciar procesos de enseñanza-aprendizaje con calidad; mientras que los estudiantes tienen un apego acelerado en el uso de las mismas. Por ello, la implementación de recursos tecnológicos promueve un cambio 
de paradigma en instituciones de educación superior, que permitirá formar seres competitivos acorde a una educación del milenio. 


\section{Bibliografía}

Alonso, C. (2001). Las nuevas funciones docentes y los nuevos medios. Facultad de Pedagogía. Universitat de Barcelona. Recuperado el 12 de diciembre de 2018, de http://dewey.uab.es/pmarques

Bordignon, F., Cicala, R., Di Salvo, C. J., Martinelli, S., \&Perazzo, M. (2011). Entornos virtuales de aprendizaje utilizados para la enseñanza en profesorados y universidades nacionales. La Plata: UNIPE.

Bautista García-Vera A. (2004). Las nuevas tecnologías en la enseñanza. Temas para el usuario. ISBN 978-84-460-2175-9. Madrid: Akal.

Cabero, J., Martínez, F., Salinas, J., (Coords.) (2003). Medios y herramientas de comunicación para la educación universitaria. En Martínez, F., El profesorado ante las nuevas tecnologías. Panamá: Sucesos Publicidad.

Cabero, J., Bartolomé, A., Cebrián, M., Duarte, A., Martínez, F., Salinas, J. (1999). Tecnología educativa. Madrid: Editorial Síntesis.

Cabero, J. y otros (1999). La formación y el perfeccionamiento del profesorado en nuevas tecnologías. En J. Ferres y P. Marqués (coords.), Comunicación educativa y nuevas tecnologías. Madrid, Praxis.

Duderstand, J. (1997). "The future of the university in an age of knowledge". Journal of Asynchronous Learning Networks (vol. 1, n.ํㅡ) 2). Sloan Consortium. Recuperado el 19 de noviembre de

García Vallinas, E. (2007). Prólogo: Empleo, formación y justicia social. Revista TAVIRA, noo 24, 2008, http://rodin.uca.es:8081/xmlui/bitstream/handle/10498/9962 /34 828916.pdf?sequen

Gonzales, B. (2012). Educación y pedagogía para el siglo XXI. Recuperado el 19 de diciembre de 2018, de: http://pedablogia.wordpress.com/2012/06/15/lascompetenciasdigitales- de-los-docentes-del-siglo-xxi/, publicado el 15 de junio de $\underline{2012 .}$

Hargreaves, A. (2000). Nueva profesionalidad para una profesión paradójica. Cuadernos de Pedagogía, N²90. Abril-2000

Ibáñez, C. (2011). Internet y su utilización por parte del profesorado en el aprendizaje de los educandos en el ciclo de educación secundaria del Liceo Experimental Manuel de Salas. Tesis doctoral. Madrid: UCM.

OCDE (2002). Organización para la Cooperación y el Desarrollo Económico, Informe. Recuperado el 23 de noviembe de 2018, de http://www.oecd.org/dataoecd/18/27/34023784.pdf.

OEI (2011). Integración TIC en la escuela, indicadores cualitativos y metodología de la investigación. Recuperado de: www.oei-idietics.org

PARCERISA, A. (1996). Materiales curriculares. Cómo elaborarlos, seleccionarlos y usarlos. Barcelona: Graó.

Salinas, J. (1997). "Nuevos ambientes de aprendizaje para una sociedad de la información". Revista Pensamiento Educativo [artículo en línea] (n. 20; pp. 81-104). PUC de Chile. 
Recuperado el 12 de noviembre de 2018, de http://www.uib.es/depart/gte/ambientes.html

UNESCO (2008). Estándares de competencias en Tic para docentes. Recuperado el 17 de diciembre de 2018, de http://portal.unesco.org/es/ev.phpURL ID=41553\&URL DO=DO TOPIC\&URL SECTION=201.html

UNESCO (2010). Metas educativas 2021: desafíos y oportunidades informe sobre tendencias sociales y educativas en América Latina 2010. Recuperado el 19 de diciembre de 2018, de http://unesdoc.unesco.org/images/0018/001899/189945s.pdf

\section{Autores}

JUAN COBOS -VELASCO obtuvo su titulo de Doctor Ph.D. En investigación Educativa emitido por el departamento de Educación de la Universidad de Alicante (España) en el 2018, obtuvo el título de MSc. En Sistemas informáticos Educativos en la universidad Tecnológica Israel en el (2005) el título de Lic. Obtuvo en la universidad Central del Ecuador en el 1996.

Actualmente de profesor titular en la Facultad de Filosofía Leras y Ciencias De La Educación en la carrera de Pedagogía de Las Ciencias Experimentales Informática, Coordinador de la catedra de Emprendimiento de la universidad central del Ecuador y es Coordinador técnico en la Revista Catedra de la Facultad de Filosofía Letras y Ciencias de la Educación sus principales temas de investigación incluyen temas relacionados competencias digitales de los Docentes, estudiantes, aplicaciones de la web aplicadas a la educación, recursos digitales para le mejora del rendimiento y el emprendimiento en la Universidad Central Ecuador , libros como de los sistemas digitales al aula, el E-learnig en la educación superior.

LILIAN JARAMILLO-NARANJO obtuvo su título de Magíster en Tecnologías para la Gestión y Práctica Docente por la Facultad de Educación de la Pontificia Universidad Católica del Ecuador (Ecuador) en 2015. Obtuvo el título de Magíster en Educación y Desarrollo Social en la Facultad de Comunicación Artes y Humanidades de la Universidad UTE (Ecuador) en 2008. Obtuvo el título de Licenciada en Biología por la Facultad de Filosofía, Letras y Ciencias de la Educación de la Universidad Central del Ecuador en 2007. Obtuvo el título de Doctoraen Ciencias de la Educación.

Actualmente es profesora titular auxiliar de la Universidad UTE del Ecuador. Es articulista y miembro del Consejo Internacional de Revisores de Revista Sophia de la Universidad Politécnica Salesiana (Ecuador). Sus principales temas de investigación incluyen educación, TIC, Biología, Ciencias Naturales, Didáctica de Ciencias Naturales, desarrollo de estrategias metacognitivas para la práctica docente desde las TIC. Ha participado en ponencias internacionales organizado por la Universidad Técnica del Norte 2018. Es autora de capítulos de libros y artículos publicados en revistas de alto impacto (Emerging Source Citation Index, Latindex, Redalcy, Scielo). Es autora del Libro Realidad Nacional para la Universidad UTE.

SANTIAGO VINUEZA-VINUEZA obtuvo su título de Magister en Redes de Comunicaciones, de la Facultad de Ingeniería, de la Pontificia Universidad Católica del Ecuador en 2016, Magíster en Sistemas Informáticos Educativos, Universidad Tecnológica Israel en 2009, Licenciado en Ciencias de la Educación especialización de Informática, Facultad de Filosofía Letras y Ciencias de la Educación, Universidad Central del Ecuador en 2002, Ingeniero en Ejecución Informática, Universidad Autónoma de Quito en 2002. 
Actualmente es profesor Agregado de la Facultad de Ciencias Económicas de la Universidad del Ecuador. Vocal Principal del Fondo de Cesantía de la Universidad Central del Ecuador, Vocal del H.C. Directivo de la Facultad de Ciencias Económicas de la Universidad Central del Ecuador, Coordinador de la Unidad de Titulación de la Carrera de Finanzas de la Universidad Central del Ecuador, Coordinador de la Asignatura de Sistemas de Información de la Facultad de Ciencias Económicas de la Universidad Central del Ecuador, Sus principales investigaciones se enmarcan en el campo educativo y las Tecnologías de la Información y Comunicación. 